\title{
QUALIDADE NUTRICIONAL E CONTAMINANTES DA BATATA (Solanum tuberosum L., SOLANACEAE) CONVENCIONAL E ORGÂNICA NA REGIÃO METROPOLITANA DE CURITIBA - PARANÁ*
}

\author{
SÔNIA CACHOEIRA STERTZ** \\ MARIA IVERLY SANTOS ROSA*** \\ RENATO JOÃO SOSSELA DE FREITAS ${ }^{* * * *}$
}

\begin{abstract}
Foram analisadas amostras de batata oriundas dos sistemas de cultivo convencional e orgânico da Região Metropolitana de Curitiba (Paraná - Brasil) em relação à sua composição nutricional e contaminantes. As batatas cultivadas pelo sistema orgânico apresentaram 19,57\% a mais de matéria seca e $138,94 \%$ a mais de açúcares, comparativamente às convencionais $(p<0,05)$. Batatas com alto teor de sólidos produzem fritas com melhor textura, menos oleosas e com melhor paladar do que as com baixo teor de sólidos. $\mathrm{O}$ alto teor de açúcares exerce influência na formação da cor dourada das fritas. As batatas orgânicas apresentaram maiores teores de $\mathrm{Fe}, \mathrm{Al}, \mathrm{Co}, \mathrm{P}, \mathrm{Se}, \mathrm{Ca}$ e $\mathrm{Cu}$, menores teores $(80 \%)$ de nitritos e nitratos quando comparadas às convencionais e ausência de resíduos de agrotóxicos. Detectou-se resíduo do organofosforado monocrotofós em uma amostra de batata convencional, em concentração $(0,13 \mathrm{mg} / \mathrm{kg})$ acima do Limite Máximo de Resíduos permitido pela legislação brasileira na época da análise da amostra $(0,05 \mathrm{mg} / \mathrm{kg})$. Conclui-se de que a batata cultivada pelo sistema orgânico oferece maior apelo em relação à segurança alimentar e à qualidade de vida.
\end{abstract}

PALAVRAS-CHAVE: AGROTÓXICOS; MINERAIS; NITRITOS E NITRATOS; BATATA (Solanum tuberosum L., SOLANACEAE).

* Parte da tese de doutorado do primeiro autor.

** Doutora, Pesquisadora do Programa de Pós-Graduação em Tecnologia de Alimentos, Setor de Tecnologia, Universidade Federal do Paraná (UFPR), Curitiba - PR - Brasil (e-mail: stertz@ufpr.br).

*** Química das Usinas Piloto de Tecnologia Química, Setor de Tecnologia da UFPR, Curitiba - PR.

Professor do Programa de Pós-Graduação em Tecnologia de Alimentos, Setor de Tecnologia, UFPR, Curitiba - PR. 


\section{INTRODUÇÃO}

No Brasil, a produção de batatas é quase que exclusivamente destinada ao consumo caseiro, com exceção de pequena produção de batatas fritas e minimamente processadas em nível industrial. As características de interesse para o processamento incluem a densidade, a textura e 0 teor de açúcares redutores. O teor de matéria seca ou sólidos totais é importante quanto ao rendimento, absorção de óleo durante a fritura e textura do produto final (PEREIRA, 1987).

Embora em declínio, a batata é considerada uma das principais hortaliças do Estado do Paraná com área de cultivo de 29.183 ha e produção de 703.749 t na safra 2003/2004 (PARANÁ, 2004). O uso de agrotóxicos e adubos químicos é intenso, nessa cultura, o que aliado ao processo de erosão do solo agrícola caracteriza forte impacto ambiental. A preocupação aumenta, pois as duas bacias hidrográficas que banham a região (Iguaçu e Ribeira) estão contaminadas por agrotóxicos há vários anos (MEDEIROS, 1984). Como medida preventiva de proteção aos mananciais, ao solo e à saúde da população pretende-se substituir, praticamente, toda a agricultura convencional por orgânica na Região Metropolitana de Curitiba (RMC).

Especialmente nos últimos sete anos, o número de produtores e a demanda por produtos orgânicos aumentaram no Paraná e na RMC. Tal fato, pode ser evidenciado pelo crescimento da produção, superior a $1400 \%$. Além disso, $50 \%$ dos 3908 produtores possui certificação e os demais encontram-se em fase de conversão dos sistemas de cultivo convencional para o orgânico (EMATER, 2004).

Estudos para comparar os alimentos obtidos pelos sistemas de cultivo convencional e orgânico em relação ao seu valor nutricional têm sido realizados. No entanto, poucos chegaram a conclusões válidas cientificamente, com exceção do conteúdo de nitrato, matéria seca, vitamina $\mathrm{C}$ e alguns antioxidantes (BOURN e PRESCOT, 2002; AFSSA, 2003). Esses dados evidenciam a necessidade de novos e criteriosos estudos.

O objetivo geral deste trabalho foi avaliar a qualidade de batatas convencionais e orgânicas produzidas na Região Metropolitana de Curitiba $(\mathrm{RMC})$ em relação às características nutricionais e contaminantes. 


\section{MATERIAL E MÉTODOS}

\subsection{COLETA E PREPARO DA AMOSTRA}

Em dezembro de 2001 foram coletadas 12 amostras de batata (var. Mona lisa) no estádio de maturação comercial e disponíveis para consumo, junto às Centrais de Abastecimento do Paraná S.A. (CEASA-PR), propriedades rurais e associações (AOPA, APAC) da RMC.

Os lotes das amostras, com cerca de 5 quilos cada, foram transportados para o laboratório de Química Analítica Aplicada/Universidade Federal do Paraná no período máximo de três horas após a coleta.

As amostras foram ligeiramente lavadas com água corrente e água destilada (para eliminar sujidades) e secas com guardanapo de papel (para retirar o excesso de umidade).

As determinações de $\mathrm{pH}$ e umidade foram efetuadas com as amostras in natura.

Para a determinação de resíduos de agrotóxicos, as amostras foram acondicionadas em sacos de polietileno, transportadas em caixas de isopor com gelo, devidamente rotuladas e levadas ao laboratório para análises. Em seguida, foram trituradas em processador de alimentos em baixa rotação (3.000 rpm) por dois minutos, congeladas em potes de plástico com boca larga, fechados com tampas também de plástico, e armazenadas em congelador a $-18^{\circ} \mathrm{C} \pm 2^{\circ} \mathrm{C}$ até o momento da análise. Foram utilizados reagentes grau pesticida (Mallinckrodt), padrões (Fluka), processador de alimentos (Wallita Mega Master Pro), balão com três bocas esmerilhadas ( $500 \mathrm{~mL}$ de capacidade), equipamento para evolução do $\mathrm{CS}_{2}$ com duas armadilhas, banho ultratermostático a 15으 (Tecnal mod TE-184), espectrofotômetro (Milton Roy - mod. Spectronic Genesys 5), Cromatógrafo a líquido (marca HP, modelo HP 1100 com detector de UV), Cromatógrafo a gás Massav (marca HP, modelo HP 5890 com detector de ECD e NPD).

Para as demais determinações, as amostras foram levadas à estufa com circulação forçada de ar (Marconi MA 035) na temperatura de $55-60^{\circ} \mathrm{C}$, durante 12 horas. Após homogeneizadas e trituradas em multiprocessador de alimentos, em baixa rotação (3000 rpm) por dois minutos, foram passadas em tamis de $2 \mathrm{~mm}$ de $\varnothing$ e acondicionadas em potes plásticos com tampa até o momento da análise. 


\subsubsection{Composição centesimal}

As proteínas foram determinadas pelo nitrogênio total, utilizando-se 0 método de Kjeldahl (macro-Kjeldahl Prodicil) e o fator 6,25 para conversão em proteína, conforme o método 955.04C da AOAC (2000). Determinouse o extrato etéreo (lipídios) por extração com éter etílico durante cinco horas em extrator de Soxhlet (Prodicil) conforme método 920.39C da AOAC (2000). As cinzas foram determinadas pela calcinação em mufla (Quimis) a $550-600^{\circ} \mathrm{C}$ durante cinco horas de acordo com o método 900.02A da AOAC (2000). Determinou-se a umidade em estufa (Marconi MA 035) com circulação forçada de ar na temperatura de 55-60을 durante 12 horas ou até peso constante, conforme método 925.10 da AOAC (2000). Os carboidratos totais foram calculados por diferença [100 g total g (proteína, lipídios, umidade, cinzas)], portanto incluindo a fração fibra alimentar (USP, 2001).

\subsubsection{Energia total metabolizável}

Calculou-se a energia procedente dos nutrientes, considerando os fatores de conversão de Atwater: $\mathrm{kcal}=(4 \times \mathrm{g}$ proteína $)+(4 \times \mathrm{g}$ carboidratos (carboidratos totais - fibra alimentar)) + (9 x g lipídios) (USP, 2001).

\subsubsection{Sólidos totais}

A matéria seca das amostras foi calculada pela diferença entre 100 e a umidade.

\subsection{4 pH}

Determinou-se o $\mathrm{pH}$ por potenciometria (Peagâmetro Orion, modelo $710^{\mathrm{A}}$ ) de acordo com o método 981.12 da AOAC (2000).

\subsubsection{Fibra alimentar}

Determinou-se a fibra alimentar utilizando a combinação de métodos enzimático e gravimétrico. As amostras secas com baixo teor de gordura (gordura $<5 \%$ ) foram gelatinizadas com $\alpha$-amilase (Termamyl $120 \mathrm{~L}$, Novo Nordisk A/S) e digeridas enzimaticamente com protease (Savinase 16 L, Novo Nordisk A/S) e amiloglugosidase (AMG 300 L, Novo Nordisk A/S) para a remoção da proteína e do amido presentes na amostra (Banho- 
maria Dubnoff Nova Ética, mod. 304). As fibras não-digeridas enzimaticamente foram precipitadas com a adição de etanol, sendo o resíduo filtrado e lavado com etanol e acetona. Após a secagem, o resíduo foi pesado. Metade das amostras foi utilizada para análise de proteínas e a outra para a análise de cinzas. O total de fibra alimentar é o peso do resíduo menos o peso das proteínas e das cinzas de acordo com o método 992.16 da AOAC (2000).

\subsubsection{Açúcares}

Glucose, sacarose, frutose e maltose foram determinadas por cromatografia a líquido de alta eficiência - CLAE (Controlador Waters 600 E Millipore; Waters 712 WISP Millipore; Detector de Índice de Refreção 1037 A HP) de acordo com o método 982.14 da AOAC (2000). Foram empregados padrões da Sigma-Aldrich para a frutose (d(-)Fructose, $0,05 \mathrm{~mol} \%$ ), maltose (4-0- $\alpha$-D-Glucopyranosyl-D-Glucose, monohidratado, 99\%), glucose (D-(+)-Glucose, Dextrose, 99,5\%) e sacarose ( $\alpha$-DGlucopyranosyl $\beta$-D-frutofuranoside, >99,5\%).

\subsubsection{Minerais}

Foram determinados os teores de alumínio, cálcio, cádmio, chumbo, cobalto, cobre, ferro, fósforo, magnésio, manganês, mercúrio, potássio, sódio, selênio e zinco, segundo o método 999.10 da AOAC (2000). As amostras foram homogeneizadas, sendo $400 \mathrm{mg}$ digeridas com $\mathrm{HNO}_{3}$ em forno de microondas (DGT 100, Provecto Sistemas Analíticos) por 15 minutos. Diluiu-se a solução com água bi-deionizada até concentração adequada para cada elemento, transferindo-a para frasco de polietileno. Procedeu-se a leitura mediante Fonte Geradora de Plasma Acoplado a Espectrômetro de Massa - ICP-MS (PE SCIEX ELAN 6000; Nebulizador do tipo "cross-flow", com "Ryton Spray Chamber"; Autosampler Perkin Elmer AS 91 acoplado a FIAS 400), com o auxílio de curva-padrão para cada elemento.

\subsubsection{Nitritos e nitratos}

Os teores de nitrito e nitrato foram determinados conforme o método $4110 \mathrm{~B}$, descrito pela APHA (1998). Pesou-se 0,5 g de amostra seca, diluiu-se em balão volumétrico de $100 \mathrm{~mL}$ com água destilada e deionizada, agitando-se ocasionalmente por uma hora. Completou-se o volume e filtrou-se a solução em papel filtro faixa preta (Whatmann 389). Procedeu-se a leitura direta em Cromatógrafo lônico de Alta Eficiência 
(HPIC), Dionex modelo DX-500, consistindo de coluna lon-Pac AS14A $(4 \times 250 \mathrm{~mm})$; pré-coluna AG-14 (4 x $50 \mathrm{~mm})$; detetor de condutividade modelo ED 40; amostrador automático modelo AS 40, observando-se as recomendações do manual do usuário da DIONEX (1999), bem como os parâmetros de calibração e validação da metodologia utilizada. As condições de operação foram: eluição isocrática; fluxo $1,2 \mathrm{~mL} /$ minuto; volume de injeção $50 \mu \mathrm{L}$; limite de deteção $0,05 \mathrm{mg} / \mathrm{L}$; limite de quantificação $0,05 \mathrm{mg} / \mathrm{L}$; e eluente, mistura de $\mathrm{Na}_{2} \mathrm{CO}_{3}$ $(8 \mathrm{mmol} / \mathrm{L})$ e $\mathrm{NaHCO}_{3}(1 \mathrm{mmol} / \mathrm{L})$.

\subsubsection{Análise de resíduos de agrotóxicos}

Os teores de multirresíduos foram quantificados por varredura em cromatografia a gás para os grupos químicos organoclorados, organofosforados, carbamatos e piretróides. Foram pesquisadas as moléculas de Aldrin, Alfa-BHC, Beta-BHC, Bifenox, Clorotalonil, Deltametrina, Dieldrin, Endossulfan I, Endossulfan II, Endossulfan sulfato, Endrin, Endrin aldeído, Fenarimol, Heptacloro, Heptacloro epóxido, Imidan, Iprodione, Lindano, Metolaclor, Metoxicloro, o,p'Dicofol, p,p'Dicofol, Propiconazol, Trifuralina, Vinclozolin, 4,4'-DDD, 4,4'-DDE, 4,4'-DDT, Clorfenvinfós, Clorpirifós, Cumafós, Demeton, Diazinon, Diclorvós, Dimetoato, Dissulfoton, Etion, Etoprofós, Fenclorfós, Fenitrotion, Fention, Forato, Fosfamidon, Metamidofós, Metidation, Mevinfós, Naled, Paration metílico, Pirazofós, Pirimifós metílico, Tocution, Triazofós, Tricloronato, Carbofuran, Carbaril, Metiocarb, Permetrina e Monocrotofós. Também foram determinados os teores de ditiocarbamatos pela quantificação do dissulfeto de carbono $\left(\mathrm{CS}_{2}\right)$ por espectrofotometria e benzimidazóis para os princípios ativos Carbendazim (methyl benzimidazol -2- ylcarbamate), Benomil [1 - (butylcarbamoyl) benzimidazol -2- ylcarbamate)] e Tiofanato metílico [dimethyl 4,4' - (o-phenylene) bis (3-thioallophanato)] por cromatografia a líquido (KEPPEL, 1971; CDFA, 1999a e 1999b).

\subsection{ANÁLISE ESTATIISTICA}

Os dados obtidos foram analisados estatisticamente pelo programa Microsoft Excel (MICROSOFT, 1997) para o cálculo das médias, desviopadrão e coeficiente de variância.

Com o auxílio do programa Minitab (MINITAB, 1998), os dados foram submetidos à análise de variância, teste $\mathrm{F}$ e Teste de Tukey para verificar a ocorrência de diferenças estatisticamente significativas $(p<0,05)$ entre os cultivos. 


\section{RESULTADOS E DISCUSSÃO}

Para FILGUEIRA (1982) e RUBATZKI e YAMAGUCHI (1997), a composição química da batata pode variar de $63 \%$ a $87 \%$ para umidade, de $13 \%$ a $37 \%$ para sólidos totais, de $13 \%$ a $30 \%$ para carboidratos totais e de $1 \%$ a $5 \%$ para proteína. Os teores obtidos no presente estudo enquadram-se nessa faixa de resultados (Tabela 1).

As amostras de batatas cultivadas organicamente apresentaram maiores teores de matéria seca $(19,57 \%)$ e açúcares $(138,94 \%)$ em relação ao sistema convencional $(p<0,05)$ (Tabela 1). O alto teor de açúcares exerce influência negativa na formação da cor dourada, sendo, porém, desejável em certos produtos. Batatas com alto teor de sólidos produzem fritas com melhor textura, menos oleosas e com melhor paladar do que as com baixo teor de sólidos (PEREIRA, 1987).

As amostras de batata orgânica apresentaram teores mais elevados de cinzas, fibras e proteínas $(68,22 \%, 20,65 \%$ e $28,70 \%$ respectivamente) que as cultivadas convencionalmente (Tabela 1), embora não tenha sido verificada diferença estatística significativa.

DLOUHY (1989) comparou durante cinco anos batatas cultivadas pelos sistemas convencional e biodinâmico, analisando os teores de matéria seca, proteína, nitrato e vitamina C. As batatas convencionais apresentaram maiores teores de proteína e nitrato que as biodinâmicas, enquanto que essas revelaram maiores teores de matéria seca e vitamina $\mathrm{C}$ que as convencionais.

Conforme o UDSA (2004) e a USP (2001) cada $100 \mathrm{~g}$ de batata contém cerca de $7 \mathrm{mg}$ de $\mathrm{Ca}, 394 \mathrm{mg}$ de K, $21 \mathrm{mg}$ de $\mathrm{Mg}, 6 \mathrm{mg}$ de Na, 0,05 mg de $\mathrm{Cu}, 0,76 \mathrm{mg}$ de Fe, 0,2 mg de $\mathrm{Mn}$ e 0,2 mg de Zn. Entretanto, para FILGUEIRA (1982) e RUBATZKI e YAMAGUCHI (1997), os compostos inorgânicos ou minerais da batata diferem muito de acordo com a variedade, tratos culturais, clima, local de plantio, maturação e armazenamento. Tal fato justifica a variabilidade nos estudos apresentados para teores de minerais quando comparados os sistemas de cultivo.

LAIRON et al. (1984) analisaram amostras de batata orgânica e convencional em relação aos teores de matéria seca, proteína, aminoácidos, nitrato, $\mathrm{P}, \mathrm{K}, \mathrm{Ca}, \mathrm{Mg}, \mathrm{Na}, \mathrm{Fe}, \mathrm{Cu}$ e Mn. Observaram que os teores de matéria seca, vitamina C, K, Ca, Fe, Cu e Mn não foram afetados pelo tipo de tratamento. Porém, verificaram tendência de maiores teores de $\mathrm{P}$ e Mg nas amostras obtidas organicamente. 


\section{TABELA 1 - DETERMINAÇÕES FÍSICO-QUÍMICAS EM AMOSTRAS DE BATATA OBTIDAS PELOS SISTEMAS DE CULTIVO CONVENCIONAL E ORGÂNICO}

\begin{tabular}{|c|c|c|c|}
\hline Determinax̧ães & Unidade & $\begin{array}{c}\text { Convencional } \\
n^{(1)}=6 \\
\text { MédatDP }^{(2)}\end{array}$ & $\begin{array}{c}\text { Orgânio } \\
n^{(1)}=6 \\
\text { Méda+DP }^{(2)}\end{array}$ \\
\hline Utidade & $\mathrm{q}$ & $8222+0,59^{2}$ & $78.74 \pm 3.01^{0}$ \\
\hline Metéria seca & g & $17,78 \pm 0,59^{b}$ & $21,26 \pm 3,01^{\mathrm{a}}$ \\
\hline anzas & $\mathrm{g}$ & $0,67 \pm 0,04^{\text {ns }}$ & $1,13+0,55^{\text {s }}$ \\
\hline Fibras & $\mathrm{g}$ & $1,99+0,31^{\text {ns }}$ & $240 \pm 0,37^{n s}$ \\
\hline Lipídos & g & $0,14 \div 0,09^{\mathrm{ss}}$ & $0,15 \pm 0,08^{\text {ns }}$ \\
\hline Proténas & $g$ & $1,32+0,70^{\text {ss }}$ & $1,70 \pm 0,16^{\text {ns }}$ \\
\hline Carboidratos & $\mathrm{g}$ & $15,65 \pm 0,70^{b}$ & $18,30+259^{a}$ \\
\hline Energia & $\mathrm{kcal}$ & $61 \pm 200^{\circ}$ & $72+10,77^{2}$ \\
\hline $\mathrm{pH}$ & & $6,47 \pm 0,18^{b}$ & $6,76+0,25^{a}$ \\
\hline Futose & $\mathrm{g}$ & $0,00 \pm 0,00^{\mathrm{s}}$ & $0,00 \pm 0,00^{\mathrm{s}}$ \\
\hline Guoose & g & $0,10 \pm 0,12^{n s}$ & $0,25 \pm 0,15^{\text {ns }}$ \\
\hline Maltose & g & $0,00 \pm 0,00^{\mathrm{ss}}$ & $0,00 \pm 0,00^{\mathrm{ns}}$ \\
\hline Suroose & $\mathrm{g}$ & $0,31 \pm 0,35^{b}$ & $0,73+0,10^{a}$ \\
\hline Soma dos aácares & g & $0,41 \pm 0,40^{b}$ & $0,98+0,24^{a}$ \\
\hline Minerais & & & \\
\hline Cálcio, Ca & $\mathrm{mg}$ & $2,49+0,70^{\mathrm{s}}$ & $291 \pm 0,46^{\mathrm{ns}}$ \\
\hline Potássio, K & $\mathrm{mg}$ & $147,90 \pm 68,92^{1 s}$ & $162,80+24,61^{\text {ns }}$ \\
\hline Magnésio, Mg & $\mathrm{mg}$ & $7,54 \pm 10,35^{\text {ns }}$ & $4,62+0,79^{1 s}$ \\
\hline Sódo, Na & $\mathrm{mg}$ & $0,10 \pm 0,26^{\text {ns }}$ & $0,00+0,00^{\text {s }}$ \\
\hline Fósforo, P & $\mathrm{mg}$ & $15,38 \pm 6,02^{1 \mathrm{~s}}$ & $20,95 \pm 6,44^{\text {ns }}$ \\
\hline Cobalto, Co & uq & $1,20+0,90^{\mathrm{s}}$ & $1,80 \pm 1,08^{n s}$ \\
\hline codre, $\mathrm{ar}$ & $\mathrm{mg}$ & $0,06+0,03^{1 s}$ & $0,07 \pm 0,0 R^{1 s}$ \\
\hline Ferro, Fe & $\mathrm{mg}$ & $0,28+0,24^{\text {ns }}$ & $0,43 \pm 0,13^{n s}$ \\
\hline Manganês, Mh & $\mathrm{mg}$ & $0,14 \pm 0,09^{\mathrm{s}}$ & $0,13 \pm 0,01^{\mathrm{ns}}$ \\
\hline Selêrio, Se & $\mu g$ & $7,47 \pm 4,41^{\text {ns }}$ & $9,9 R+4,0 R^{n s}$ \\
\hline Znoo, Zn & $\mathrm{mg}$ & $0,18+0,10^{\mathrm{s}}$ & $0,17 \pm 0,0 \mathrm{~B}^{\mathrm{ns}}$ \\
\hline Metais pesados & & & \\
\hline Alumínio, A & $\mathrm{mg}$ & $0,30 \pm 0,36^{n s}$ & $0,46 \pm 0,15^{\mathrm{ns}}$ \\
\hline Cádrio, Cd & $\mu \mathrm{q}$ & $1,65+0,06^{n s}$ & $0,21 \pm 0,39^{\text {s }}$ \\
\hline Merário, $\mathrm{Hg}$ & $\mu \mathrm{g}$ & $0,40 \pm 0,25^{\text {ns }}$ & $0,74 \pm 0,47^{n s}$ \\
\hline Chumbo, Po & $\mu g$ & $5,95 \pm 3,49^{\mathrm{s}}$ & $3,94 \pm 1,24^{\text {ns }}$ \\
\hline
\end{tabular}

Médias seguidas na mesma linha por letras iguais não diferem estatisticamente no nível de $5 \%(p<0,05)$.

ns $=$ não-significativo.

Os resultados correspondem à média das amostras, analisadas em triplicata.

(1) $n$ = número de amostras analisadas;

(2) Valores por $100 \mathrm{~g}$ de amostra, expressos em base úmida. 
HANSEN (1981) comparou batatas e beterrabas cultivadas pelos sistemas convencional (quatro propriedades) e biodinâmico (quatro propriedades) em diferentes regiões, mas com tipo de solo e clima similares. Em três das quatro regiões não observou maiores diferenças em relação aos teores de $\mathrm{N}$, cinzas, $\mathrm{P}, \mathrm{K}, \mathrm{Na}$, $\mathrm{Ca}$ e $\mathrm{Mg}$, com exceção de menores níveis de nitrato nas beterrabas biodinâmicas.

A batata orgânica apresentou menores teores de nitritos e nitratos (cerca de $80 \%)$ e maiores teores de $\mathrm{Fe}(53,57 \%)$, Al $(53,33 \%)$, Co $(50,20 \%), \mathrm{P}$ $(36,24 \%)$, Se (32,74\%), Ca (16,81\%) e Cu (16,67\%) quando comparada com a convencional (Tabela 1 e 2). Tal tendência também foi constatada por BOURN e PRESCOT (2002) e AFSSA (2003).

\section{TABELA 2 - TEORES DE NITRITOS ( $\left.\mathrm{NO}_{2}^{-}\right)$E NITRATOS $\left(\mathrm{NO}_{3}^{-}\right)$EM BATATAS OBTIDAS PELOS SISTEMAS DE CULTIVO CONVENCIONAL E ORGÂNICO NA RMC}

\begin{tabular}{|c|c|c|}
\hline \multirow[t]{2}{*}{ Determinações* } & $\begin{array}{c}\text { Convencional } \\
n=6\end{array}$ & $\begin{array}{c}\text { Orgânico } \\
n=6\end{array}$ \\
\hline & Média+DP & Média+DP \\
\hline $\begin{array}{l}\mathrm{NO}_{2}^{-} \\
\mathrm{NO}_{3}^{-} \\
\mathrm{S}\end{array}$ & $\begin{array}{c}3,03 \pm 3,37^{1 \mathrm{~s}} \\
72,80 \pm 140,20^{\text {ns }} \\
75,83 \pm 138,72^{\text {ns }}\end{array}$ & $\begin{array}{c}0,00 \pm 0,00^{n s} \\
15,52+5,83^{n s} \\
15,52 \pm 5,83^{\text {ns }}\end{array}$ \\
\hline
\end{tabular}

$\mathrm{S}=$ soma dos teores de nitritos e nitratos; $\mathrm{n}=$ número de amostras analisadas; $\mathrm{DP}=$ desvio-padrão; *valores apresentados em base úmida, em $\mathrm{mg} / \mathrm{kg}$; $\mathrm{ns}$ = nãosignificativo ao nível de $5 \%(p<0,05)$.

LYONS et al. (1994), XIMENES, RATH e REYES (2000), YORDANOV, NOVAKOVA e LUBENOVA (2001) compararam hortícolas (espinafre, agrião, alface, batata, tomate e cenoura) cultivadas pelos sistemas convencional e orgânico, tratadas com diferentes tipos de fertilizantes em relação aos teores de nitrogênio e nitrato. Tais estudos não mostraram diferenças estatisticamente significativas entre os sistemas, devido à considerável variabilidade de valores encontrados. Entretanto, outros autores apontaram níveis mais baixos de nitratos para os produtos orgânicos, principalmente nas culturas de alface, batata, cenoura e espinafre (LECLERC et al., 1991; VOGTMANN et al., 1993; RAUPP, 1998; 
BOURN e PRESCOT, 2002). Contudo, a comparação dos sistemas de cultivo pode ser considerada apenas para as condições do estudo, visto a diversidade e complexidade de fatores que influenciam a absorção do nitrogênio pelas plantas e sua conseqüente transformação em nitritos e nitratos (HORNICK, 1992; BOURN e PRESCOT, 2002; AFSSA, 2003).

Enquanto as amostras advindas do sistema de cultivo orgânico apresentaram total ausência de resíduos de agrotóxicos foi detectado resíduo do organofosforado (OF) monocrotofós (dimethyl (E)-1-methyl-2(methylcarbamoyl)vinyl phosphate) em uma amostra de batata convencional. A concentração de $0,13 \mathrm{mg} / \mathrm{kg}$ verificada situou-se acima do Limite Máximo de Resíduos (LMR) permitido pela legislação brasileira na época da coleta e análise da amostra ${ }^{1}(0,05 \mathrm{mg} / \mathrm{kg})$. O monocrotofós, inseticida e acaricida classificado toxicologicamente como Classe I, é considerado altamente tóxico para o ser humano. Nesse período, o produto contava com registro para aplicação por pulverização nas partes aéreas das culturas de amendoim, feijão, trigo, algodão, batata e soja, com carência de 9 a 21 dias, de modo que o resíduo encontrado na batata pode ser considerado como "não-conformidade" (BRASIL, 2001).

O maior benefício do alimento obtido pelo sistema de cultivo orgânico advém de práticas culturais que melhoram o solo, oferecendo mínimo impacto ambiental. A agricultura convencional pode até produzir alimento mais barato, porém os custos externos (degradação do meio ambiente, riscos para a saúde e problemas sociais) são altos (BOURN e PRESCOTT, 2002).

\section{CONCLUSÃO}

Além de melhores características nutricionais (maiores teores de matéria seca, carboidratos, energia, açúcares), a batata orgânica apresentou menores teores de nitrito e nitrato, estando isenta de qualquer tipo de resíduo de agrotóxico. Desta forma, a batata cultivada pelo sistema orgânico oferece maior apelo em relação à segurança alimentar e à qualidade de vida.

\footnotetext{
${ }^{1} \mathrm{Na}$ época da coleta, o ingrediente ativo monocrotofós encontrava-se sob reavaliação técnica. A Resolução nํㅜ 135, de 17/05/02, republicada no DOU de 22/05/2003 e alterada pela Resolução no 98 de 05/05/2003 limitou seu emprego para as partes aéreas de algodão, soja e trigo com carência de 21 dias (BRASIL, 2004).
} 


\begin{abstract}
NUTRITIONAL QUALITY AND CONTAMINANTS OF CONVENTIONAL AND ORGANIC POTATO (Solanum tuberosum L., SOLANACEAE) IN THE METROPOLITAN REGION OF CURITIBA - PARANÁ - BRAZIL

Potato samples from conventional and organic cultivation systems of the metropolitan region of Curitiba (Parana - Brazil) were analysed, regarding their nutritional composition and contaminants. The organic potatoes showed $19.57 \%$ more dry matter and $138.94 \%$ more sugar, when compared in relation to the conventional cultivation system $(p \leqslant 0.05)$. Potatoes with high drift of sodium produced fries with a better texture, less oily and with a better taste when compared to the potatoes which showed a low drift of solids. The high drift of sugar inflluences in the formation of the golden colour of the fries. The organic potatoes showed higher drifts of $\mathrm{Fe}, \mathrm{Al}, \mathrm{Co}, \mathrm{P}, \mathrm{Ca}$ and $\mathrm{Cu}$ and lower drifts $(80 \%)$ of nitrites and nitrates when compared to the conventional cultivation system and absence of pesticide residues. It was found monocrotophos organophosphorus residues in a conventional potato sample, in a $0.13 \mathrm{mg} / \mathrm{kg}$ concentration level, therefore, above the Maximum Limit of Residues permitted by the Brazilian legislation at the time of the sample analysis, which was of $0.05 \mathrm{mg} / \mathrm{kg}$. It was concluded that the potato cultivated by the organic system offers more appeal in relation to food safety and health quality.
\end{abstract}

KEY-WORDS: PESTICIDES; MINERALS; NITRITES AND NITRATES; POTATO (Solanum tuberosum L., SOLANACEAE).

\title{
REFERÊNCIAS
}

1 AFSSA. Agence Française de Securite Sanitaire des Aliments. Evaluation nutritionnelle et sanitaire dês aliments issus de lágriculture biologique. Disponível em: <http://www.afssa.fr.htm> Acesso em: 10 out. 2003.

2 AOAC. Association of Official Analytical Chemists. Official methods of analysis of AOAC International. 17 $7^{\text {th }}$ ed. Gaithersburg, 2000. v. 1-2.

3 APHA. American Public Health Association. Standard Methods for the Examination of Water and Wastewater. $20^{\text {th }}$ ed. Washington, 1998.

4 BOURN, D.; PRESCOTT, J. A comparison of the nutritional value, sensory qualities, and food safety of organically and a conventionally produced foods. Critical Reviews in Food Science and Nutrition, Dunedin, New Zealand, v.42, n.1, p.1-34, 2002.

5 BRASIL. Agência Nacional de Vigilância Sanitária, Ministério da Saúde. Monografias de produtos agrotóxicos. Disponível em: 
<http://www.anvisa.gov.br/alimentos/tox/mono/index.htm>. Acesso em: 01 out. 2004.

6 CDFA. California Department of Food and Agriculture. Multiresidue screen for pesticides in fruits and vegetables. $3^{\text {rd }}$ ed. Sacramento, CA, 1999a.

7 CDFA. California Department of Food and Agriculture. Benomyl, Carbendazin and Thiophanate-methyl analysis. $3^{\text {rd }}$ ed. Sacramento, CA, 1999b.

8 DIONEX Corporation. AS14A manual. Sunnyvale, CA, 1999. 34 p. (Doc. oㅡ 031678-01).

9 DLOUHY, J. Product quality in alternative agriculture. In: COLLOQUIUM IN FOOD QUALITY CONCEPTS AND METHODOLOGY, 1989, Kassel. Proceedings... Kassel: Elm Farm Research Centre/University of Kassel, 1989. p.30-35.

10 EMATER. Empresa Paranaense de Assistência Técnica e Extensão Rural. Produção de alimentos orgânicos no Paraná. Disponível em: <http://www.emater.pr.br>. Acesso em: 11 de out. 2004.

11 FILGUEIRA, F.A.R. Manual de olericultura: cultura e comercialização de hortícolas. 2.ed. São Paulo: Agronômica Ceres, 1982. 357 p. (Olericultura Especial, 2).

12 FURLANI, A.M.C.; FURLANI,P.R.; BATAGLIA, O.C.; HIROCE, R.; GALLO, J.R. Composição mineral de diversas hortaliças. Bragantia, v.37, n.5, p.33-44, 1978.

13 HANSEN, H. Comparison of chemical composition and taste of biodynamically and conventionally grown vegetables. Qual. Plant. PI. Fds Hum. Nutr., v.30, p.203-211, 1981.

14 HORNICK, S. B. Factors affecting the nutritional quality of crops. Am. J. Altern. Agric., v.7, p.63-68, 1992.

15 KEPPEL, G. E. Collaborative study of the determination of dithiocarbamate residues by a modified carbon disulfide evolution method. Journal of the Association of Official Analytical Chemists, Washington, v.54, n.3. p.528-532, 1971.

16 LAIRON, D.; TERMINE, E.; GAUTIER, S.; TROUILLOUD, M.; LAFONT, H.; HAUTON, J.C. Effects of organic and mineral fertilizations on the contents of vegetables in minerals, vitamin $\mathrm{C}$ and nitrates. In: 
VOGTMANN, H.; BOEHNCKE, E.; FRICKE, I. (Ed.) The Importance of Biological Agriculture in a World of Diminishing Resources. Witzenhausen, Germany: Verlagsgruppe Weiland, 1984. p.249-260.

17 LECLERC, J.; MILLER, M.L.; JOLIET, E.; ROCQUELIN, G. Vitamin and mineral contents of carrot and celeriac under mineral or organic fertilization. Biol. Agric. Hort. v.7, p.349-361, 1991.

18 LYONS, D. J.; RAYMENT, G. E.; NOBBS, P. E.; MCCALLUM, L. E. Nitrate and nitrite in fresh vegetables from Queensland. J. Sci. Food Agric., Great Britain, v. 64, n. 3, p. 279-281, 1994.

19 MEDEIROS, M.C.M.B.; VIANNA, P.C.G.; FOWLER, R.B.; ZAPPIA, V.R.S. Poluição das águas internas do Paraná por agrotóxicos. Curitiba: SUREHMA/PR, 1984.

20 MICROSOFT Corporation. MSOFFICE Microsoft Excel 97. Redmond, WA, 1995-1997. (CDROM 6 MB).

21 MINITAB Inc. Reference manual release 7. 13 Demo. Statistical Software 13.0. Enterprise Drive, State Collegey PA, 16801 US, 1998.

22 PARANÁ. SEAB/DERAL. Hortaliças Paraná: evolução da área colhida e da produção obtida - 03/04. Disponível em: <http//www.pr.gov.br/ seab/deral/ehpr.xls>. Acesso em: 11 de out. 2004.

23 PEREIRA, A.S. Composição química, valor nutricional e industrialização. In: REIFSCHEEIDER, F.J.B. Produção de batata. Brasília: Linha Gráfica, 1987. p.12-28.

24 RAUPP, J. Examination of some microbiological and biochemical parameters and tests of product quality used in a long-term fertilization trial, Am. J. Altern. Agric., v.13, p.138-44, 1998.

25 RUBATSKY, V.E.; YAMAGUCHI, M. World Vegetables: principles, production and nutritive values., $2^{\text {nd }}$ ed. California: Chapman e Hall, 1997. p.532-536.

26 USDA. United States Department of Agriculture. Food and Nutrition Information Center, 1997. Disponível em: <http://www.nal.usda.gov/ fnic/foodcomp/data>. Acesso em: 07 out. 2004.

27 USP. Universidade de São Paulo. Tabela brasileira de composição de alimentos: projeto integrado de composição de alimentos. Disponível em: <http://www.fcf.usp.br/tabela/tbcacoce.php>. Acesso em: 10 dez. 2001. 
28 VOGTMANN, H.; MATTHIES, K.; KEHRES, B.; MEIER-PLOEGER, A. Enhanced food quality: effects of composts on the quality of plant foods. Compost Sci. And Utilization, v.1, p.82-100, 1993.

29 XIMENES, M.I.N; RATH, S.; REYES, F.G.R. Polarographic determination of nitrate in vegetables. Talanta, v.51, p.49-56, 2000.

30 YORDANOV, N.D.; NOVAKOVA, E.; LUBENOVA, S. Consecutive estimation of nitrite and nitrate íons in vegetables and fruits by electron paramagnetic resonance spectrometry. Analytica Chimica Acta, v.437, p.131-138, 2001.

\section{AGRADECIMENTOS}

Os autores agradecem o apoio financeiro fornecido pela Fundação AraucáriaPR - Projeto 567. 